\title{
Unified Treatment of the Krätzel Transformation for Generalized Functions
}

\author{
S. K. Q. Al-Omari ${ }^{1}$ and A. Kılıçman ${ }^{2}$ \\ ${ }^{1}$ Department of Applied Sciences, Faculty of Engineering Technology, Al-Balqa Applied University, Amman 11134, Jordan \\ ${ }^{2}$ Department of Mathematics and Institute of Mathematical Research, Universiti Putra Malaysia (UPM), \\ 43400 Serdang, Selangor, Malaysia \\ Correspondence should be addressed to A. K1lı̧̧man; akilicman@putra.upm.edu.my
}

Received 26 March 2013; Accepted 18 April 2013

Academic Editor: Mustafa Bayram

Copyright (C) 2013 S. K. Q. Al-Omari and A. Kılıçman. This is an open access article distributed under the Creative Commons Attribution License, which permits unrestricted use, distribution, and reproduction in any medium, provided the original work is properly cited.

We discuss a generalization of the Krätzel transforms on certain spaces of ultradistributions. We have proved that the Krätzel transform of an ultradifferentiable function is an ultradifferentiable function and satisfies its Parseval's inequality. We also provide a complete reading of the transform constructing two desired spaces of Boehmians. Some other properties of convergence and continuity conditions and its inverse are also discussed in some detail.

\section{Introduction}

Krätzel, in [1,2], introduced a generalization of the Meijer transform by the integral:

$$
\left(K_{v}^{p} f\right)(x)=\int_{\mathbf{R}_{+}} \mathbf{Z}_{p}^{v}(x y) f(y) d y, \quad x>0,
$$

where

$$
\mathrm{Z}_{p}^{v}(x y)=\int_{\mathbf{R}_{+}} t^{\nu-1} e^{-t^{p}-x y / t} d t
$$

$p>0(\in \mathbf{N}), v \in \mathbb{C}$. Then this generalization is known as Krätzel transform.

Let $x$ be in $\mathbf{R}_{+}$. Denote by $\mathcal{S}_{+}$, or $\mathcal{S}\left(\mathbf{R}_{+}\right)$, the space of all complex-valued smooth functions $\phi(t)$ on $\mathbf{R}_{+}$such that

$$
\sup _{x \in \mathbf{K}}\left|D^{k} \phi(x)\right|<\infty,
$$

$x \in \mathbf{R}_{+}$, where $\mathbf{K}$ runs through compact subsets of $\mathbf{R}_{+}$; see [3]. The strong dual $\mathcal{S}_{+}^{\prime}$ of $\mathcal{S}_{+}$consists of distributions of compact supports.

Later, the authors in [4] have studied the $K_{v}^{p}$ transformation in a space of distributions of compact support inspired by known kernel method. They, also, have obtained its properties of analyticity and boundedness and have established its inversion theorem. In the sense of classical theory, the Meijer transformation and the Laplace transformation in [5] are presented as special forms of the cited transform for $p=1$ and $p=1, v= \pm 1 / 2$, respectively.

It is worth mentioning in this note that a suitable motivation of the cited transform has thoroughly been discussed in [6] by the aid of a Fréchet space of constituted functions of infinitely differentiable functions over $(0, \infty)$.

This paper is a continuation of the work obtained in [4]. We are concerned with a general study of the transform in the space of ultradistributions and further discuss its extension to Boehmian spaces in some detail. We are employing the adjoint method and method of kernels for our purpose to extend the classical integral transform to generalized functions and hence ultradistributions.

\section{Ultradistributions}

The theory of ultradistributions is one of generalizations of the theory of Schwartz distributions; see [3, 7]. Since then, in the recent past and even earlier, it was extensively studied by many authors such as Roumieu [8, 9], Komatsu [10], Beurling [11], Carmichael et al. [12], Pathak [13, 14], and Al-Omari [15, $16]$. 
By an ultradifferentiable function we mean an infinitely smooth function whose derivatives satisfy certain growth conditions as the order of the derivatives increases. Unlike sequences presented in $[15,16], a_{i}, i=0,1, \ldots$, wherever it appears, denotes a sequence of positive real numbers. Such omission of constraints may ease the analysis.

Let $\alpha$ be a real number but fixed and $\mathscr{L}^{r}$ be the space of Lebesgue integrable functions on $\mathbf{R}_{+}$. Denote by $\mathcal{S}_{+}\left(\mathscr{L}^{r}, \alpha,\left(a_{i}\right), a\right)$ (resp., $\left.\mathcal{S}_{+}\left(\mathscr{L}^{r}, \alpha,\left\{a_{i}\right\}, a\right)\right), 1 \leq r \leq \infty$, the subsets of $\mathcal{S}_{+}$of all complex valued infinitely smooth functions on $\mathbf{R}_{+}$such that, for some constant $m_{1}(>0)$,

$$
\sup _{\alpha, x \in \mathbf{K}}\left\|\mathscr{D}^{k} \varphi(x)\right\|_{\mathscr{L}^{r}} \leq m_{1} a^{\alpha} a_{\alpha}
$$

for all $a>0$ (for some $a>0$ ), where $\mathbf{K}$ is a compact set traverses $\mathbf{R}_{+}$.

The elements of the dual spaces, $\mathcal{S}_{+}^{\prime}\left(\mathscr{L}^{r}, \alpha,\left(a_{i}\right), a\right)\left(\mathcal{S}_{+}^{\prime}\left(\mathscr{L}^{r}, \alpha,\left\{a_{i}\right\}, a\right)\right)$, are the Beurlingtype (Roumieu-type) ultradistributions. It may be noted that $\mathcal{S}_{+}\left(\mathscr{L}^{r}, \alpha,\left(a_{i}\right), a\right) \subset \mathcal{S}_{+}\left(\mathscr{L}^{r}, \alpha,\left\{a_{i}\right\}, a\right) \subset \mathcal{S}_{+}$. Thus, every distribution of compact support is an ultradistribution of Roumieu type and further, and an ultradistribution of Roumieu type is of Beurling-type. Natural topologies on $\mathcal{S}_{+}\left(\mathscr{L}^{r}, \alpha,\left(a_{i}\right), a\right)$ (resp., $\left.\mathcal{S}_{+}\left(\mathscr{L}^{r}, \alpha,\left\{a_{i}\right\}, a\right)\right)$ can be generated by the collection of seminorms:

$$
\|\varphi\|_{r, a}=\sup _{\alpha, x \in \mathbf{K}} \frac{\left\|\mathscr{D}^{k} \varphi(x)\right\|_{\mathscr{L}^{r}}}{a^{\alpha} a_{\alpha}}, \quad a>0 .
$$

A sequence $\left(\varphi_{n}\right) \rightarrow \varphi \in \mathcal{S}_{+}\left(\mathscr{L}^{r}, \alpha,\left(a_{i}\right), a\right)$ (resp., $\left.\mathcal{S}_{+}\left(\mathscr{L}^{r}, \alpha,\left\{a_{i}\right\}, a\right)\right)$ if

$$
\lim _{n \rightarrow \infty} \sup _{\alpha, x \in \mathbf{K}}\left\|\mathscr{D}^{k}\left(\varphi_{n}(x)-\varphi(x)\right)\right\|_{\mathscr{L}^{r}}=0
$$

and there is a constant $m_{1}>0$ independent of $n$ such that

$$
\lim _{n \rightarrow \infty} \sup _{\alpha, x \in \mathbf{K}}\left\|\mathscr{D}^{k}\left(\varphi_{n}(x)-\varphi(x)\right)\right\|_{\mathscr{L}^{r}} \leq m_{1} a^{\alpha} a_{\alpha}
$$

for all $a>0 . \mathcal{S}_{+}\left(\mathscr{L}^{r}, \alpha,\left(a_{i}\right), a\right)\left(\mathcal{S}_{+}\left(\mathscr{L}^{r}, \alpha,\left\{a_{i}\right\}, a\right)\right)$ is dense in $\mathcal{S}_{+}$, convergence in $\mathcal{S}_{+}\left(\mathscr{L}^{r}, \alpha,\left(a_{i}\right), a\right)\left(\mathcal{S}_{+}\left(\mathscr{L}^{r}, \alpha,\left\{a_{i}\right\}, a\right)\right)$ implies convergence in $\mathcal{S}_{+}$, and consequently a restriction of any $f \in \mathcal{S}_{+}^{\prime}$ to $\mathcal{S}_{+}\left(\mathscr{L}^{r}, \alpha,\left(a_{i}\right), a\right)\left(\mathcal{S}_{+}\left(\mathscr{L}^{r}, \alpha,\left\{a_{i}\right\}, a\right)\right)$ is in $\mathcal{S}_{+}^{\prime}\left(\mathscr{L}^{r}, \alpha,\left(a_{i}\right), a\right)\left(\mathcal{S}_{+}^{\prime}\left(\mathscr{L}^{r}, \alpha,\left\{a_{i}\right\}, a\right)\right)$.

\section{The Krätzel Transform of Tempered Ultradistributions}

In this section of this paper we define the Krätzel transform of tempered ultradistributions by using both of kernel and adjoint methods. We restrict our investigation to the case of Beurling type since the other investigation for the Roumieutype tempered ultradistributions is almost similar.

Lemma 1. Let $\phi \in \mathcal{S}_{+}\left(\mathscr{L}^{r}, \alpha,\left(a_{i}\right), a\right)$ and then $K_{v}^{p} \phi \in$ $\mathcal{S}_{+}\left(\mathscr{L}^{r}, \alpha,\left(a_{i}\right), a\right)$.
Proof. Let $\phi \in \mathcal{S}_{+}\left(\mathscr{L}^{r}, \alpha,\left(a_{i}\right), a\right)$ and $x$ be fixed, and then $K_{v}^{p} \phi$ certainly exists. By differentiation with respect to $x$ we get

$$
\begin{aligned}
\mathscr{D}_{x}\left(K_{v}^{p} \phi\right)(x) & =\iint_{\mathbf{R}_{+}} \mathscr{D}_{x} t^{v-1} e^{-t^{p}-x y / t} d t \phi(y) d y \\
& =\iint_{\mathbf{R}_{+}} \frac{-y}{t} t^{v-1} e^{-t^{p}-x y / t} d t \phi(y) d y \\
& =\int_{\mathbf{R}_{+}}-y \int_{\mathbf{R}_{+}} t^{(v-1)-1} e^{-t^{p}-x y / t} d t \phi(y) d y \\
& =\int_{\mathbf{R}_{+}}-y \mathrm{Z}_{p}^{v-1}(x y) \phi(y) d y .
\end{aligned}
$$

Hence the principle of mathematical induction on the $k$ th derivative gives

$$
\mathscr{D}_{x}^{k}\left(K_{v}^{p} \phi\right)(x)=(-1)^{k} \int_{\mathbf{R}_{+}} \mathrm{Z}_{p}^{v-k}(x y) y^{k} \phi(y) d y .
$$

From [4], we deduce that

$$
\begin{aligned}
& \left|\mathscr{D}_{x}^{k}\left(K_{v}^{p} \phi\right)(x)\right| \\
& \quad \leq \alpha_{1} \int_{\mathbf{R}_{+}}\left|(y)^{(2 v-p) /(2 p+2)} e^{-(x y)^{p} /(p+1)}\right| y^{k} \phi(y) d y
\end{aligned}
$$

for some constant $\alpha_{1}$. The assumption that $\phi \in$ $\mathcal{S}_{+}\left(\mathscr{L}^{r}, \alpha,\left(a_{i}\right), a\right)$ implies that the products under the integral sign, $\Psi=y^{k} \phi(y)$ and $(x y)^{(2 v-p) /(2 p+2)} e^{-(x y)^{p} /(p+1)} \Psi$, are also in $\mathcal{S}_{+}\left(\mathscr{L}^{r}, \alpha,\left(a_{i}\right), a\right)$. Moreover, $\phi \in \mathcal{S}_{+}\left(\mathscr{L}^{r}, \alpha,\left(a_{i}\right), a\right)$ ensures that the integral:

$$
F(x)=\alpha_{1} \int_{\mathbf{R}_{+}}\left|(x y)^{(2 v-p) /(2 p+2)} e^{-(x y)^{p} /(p+1)}\right| y^{k} \phi(y) d y
$$

belongs to $\mathcal{S}_{+}\left(\mathscr{L}^{r}, \alpha,\left(a_{i}\right), a\right)$. Hence

$$
\left\|\mathscr{D}_{x}^{k} F(x)\right\|_{\mathscr{L}^{r}} \leq m_{1} a^{\alpha} a_{\alpha}
$$

for some constant $m_{1}$. Therefore, from the above inequality we get

$$
\left\|\mathscr{D}_{x}^{k}\left(K_{v}^{p} \phi\right)(x)\right\|_{\mathscr{L}^{r}} \leq\left\|\mathscr{D}_{x}^{k} F(x)\right\|_{\mathscr{L}^{r}} \leq m_{1} a^{\alpha} a_{\alpha}
$$

for certain positive constant $m_{1}$. This proves the lemma.

From Lemma 1 we deduce that the Krätzel transform is bounded and closed from $\mathcal{S}_{+}\left(\mathscr{L}^{r}, \alpha,\left(a_{i}\right), a\right)$ into itself. Next, we establish the Parseval's relation for the Krätzel transform.

Theorem 2. Let $f$ and $g$ be absolutely integrable functions over $\mathbf{R}_{+}$and then

$$
\int_{\mathbf{R}_{+}} f(x)\left(K_{v}^{p} g\right)(x) d x=\int_{\mathbf{R}_{+}}\left(K_{v}^{p} f\right)(x) g(x) d x,
$$

where $K_{v}^{p} f$ and $K_{v}^{p} g$ are the Krätzel transforms of $f$ and $g$, respectively. 
Proof. It is clear that $K_{v}^{p} f$ and $K_{v}^{p} g$ are continuous and bounded on $\mathbf{R}_{+}$. Moreover, the Fubini's theorem allows us to interchange the order of integration:

$$
\begin{aligned}
\int_{\mathbf{R}_{+}} f(x)\left(K_{v}^{p} g\right)(x) d x \\
\quad=\int_{\mathbf{R}_{+}}\left(\int_{\mathbf{R}_{+}} f(x) \mathrm{Z}_{p}^{v}(x y) d x\right) g(y) d y .
\end{aligned}
$$

Equation (15) follows since the Krätzel kernel $\mathrm{Z}_{p}^{v}(x y)$ applies for the functions $f$ and $g$, when the order of integration is interchanged. This completes the proof of the theorem.

Now, in consideration of Theorem 2, the adjoint method of extending the Krätzel transform can be read as

$$
\left\langle K_{v}^{p} f, \phi\right\rangle=\left\langle f, K_{v}^{p} \phi\right\rangle
$$

where $f \in \mathcal{S}_{+}^{\prime}\left(\mathscr{L}^{r}, \alpha,\left(a_{i}\right), a\right)$ and $\phi \in \mathcal{S}_{+}\left(\mathscr{L}^{r}, \alpha,\left(a_{i}\right), a\right)$.

Theorem 3. Given that $f \in \mathcal{S}_{+}^{\prime}\left(\mathscr{L}^{r}, \alpha,\left(a_{i}\right), a\right)$ then $K_{v}^{p} f \in$ $\mathcal{S}_{+}^{\prime}\left(\mathscr{L}^{r}, \alpha,\left(a_{i}\right), a\right)$.

Proof. Consider a zero convergent sequence $\left(\phi_{n}\right)$ in $\mathcal{S}_{+}\left(\mathscr{L}^{r}, \alpha,\left(a_{i}\right), a\right)$ then certainly $\left(K_{v}^{p} \phi_{n}\right)$ is a zero-convergent sequence in the same space. It follows from (16) that

$$
\left\langle K_{v}^{p} f, \phi_{n}\right\rangle=\left\langle f, K_{v}^{p} \phi_{n}\right\rangle \longrightarrow 0 \text { as } n \longrightarrow \infty \text {. }
$$

Linearity is obvious. This completes the proof.

From the above theorem we deduce that the Krätzel transform of a tempered ultradistribution is a tempered ultradistribution. Moreover, the boundedness property of $K_{v}^{p} f$, $f \in \mathcal{S}_{+}^{\prime}\left(\mathscr{L}^{r}, \alpha,\left(a_{i}\right), a\right)$ follows from the following theorem.

Theorem 4. Let $f \in S_{+}^{\prime}\left(\mathscr{L}^{r}, \alpha,\left(a_{i}\right), a\right)$ and then $K_{v}^{p} f$ is bounded.

Proof. See [4, Proposition 2.3].

It is interesting to know that the Krätzel transform can be defined in an alternative way, namely, by the kernel method. Let $f \in \mathcal{S}_{+}^{\prime}\left(\mathscr{L}^{r}, \alpha,\left(a_{i}\right), a\right)$, and then

$$
\left(K_{v}^{p} f\right)(x)=\left\langle f(y), z_{p}^{v}(x y)\right\rangle
$$

In fact, (18) is a straightforward consequence of Lemma 1.

Theorem 5. Let $f \in \mathcal{S}_{+}^{\prime}\left(\mathscr{L}^{r}, \alpha,\left(a_{i}\right), a\right)$ then $K_{v}^{p} f$ is infinitely differentiable and

$$
\mathscr{D}_{x}^{k}\left(K_{v}^{p} f\right)(x)=\left\langle f(t), \mathscr{D}_{x}^{k} \mathrm{Z}_{p}^{v}(x t)\right\rangle
$$

for every $k \in \mathbf{N}$ and $x \in \mathbf{R}_{+}$.

Proof. See [4, Proposition 2.2].

\section{Boehmian Spaces}

Boehmians were first constructed as a generalization of regular Mikusinski operators [17]. The minimal structure necessary for the construction of Boehmians consists of the following elements:

(i) a nonempty set $A$,

(ii) a commutative semigroup $(B, *)$,

(iii) an aperation $\odot: \mathrm{A} \times \mathrm{B} \rightarrow \mathrm{A}$ such that for each $x \in \mathrm{A}$ and $s_{1}, s_{2} \in \mathrm{B}, x \odot\left(s_{1} * s_{2}\right)=\left(x \odot s_{1}\right) \odot s_{2}$,

(iv) a collection $\Delta \subset \mathrm{B}^{N}$ such that

(a) if $x, y \in \mathrm{A},\left(s_{n}\right) \in \Delta, x \odot s_{n}=y \odot s_{n}$ for all $n$, then $x=y$,

(b) if $\left(s_{n}\right),\left(t_{n}\right) \in \Delta$, then $\left(s_{n} * t_{n}\right) \in \Delta$.

Elements of $\Delta$ are called delta sequences. Consider

$$
\mathrm{g}=\left\{\left(x_{n}, s_{n}\right): x_{n} \in \mathrm{A},\left(s_{n}\right) \in \Delta, x_{n} \odot s_{m}=x_{m} \odot s_{n}, \forall m, n \in \mathbf{N}\right\} \text {. }
$$

If $\left(x_{n}, s_{n}\right),\left(y_{n}, t_{n}\right) \in \mathrm{g}, x_{n} \odot t_{m}=y_{m} \odot s_{n}$, for all $m, n \in \mathbf{N}$, then we say $\left(x_{n}, s_{n}\right) \sim\left(y_{n}, t_{n}\right)$. The relation $\sim$ is an equivalence relation in $g$. The space of equivalence classes in $g$ is denoted by $\beta$. Elements of $\beta$ are called Boehmians. Between $A$ and $\beta$ there is a canonical embedding expressed as

$$
x \longrightarrow \frac{x \odot s_{n}}{s_{n}} .
$$

The operation $\odot$ can be extended to $\beta \times \mathrm{A}$ by

$$
\frac{x_{n}}{s_{n}} \odot t=\frac{x_{n} \odot t}{s_{n}} .
$$

In $\beta$, there are two types of convergence:

( $\delta$ convergence) a sequence $\left(h_{n}\right)$ in $\beta$ is said to be $\delta$ convergent to $h$ in $\beta$, denoted by $h_{n} \stackrel{\delta}{\rightarrow} h$, if there exists a delta sequence $\left(s_{n}\right)$ such that $\left(h_{n} \odot s_{n}\right),\left(h \odot s_{n}\right) \in$ A, for all $k, n \in \mathbf{N}$, and $\left(h_{n} \odot s_{k}\right) \rightarrow\left(h \odot s_{k}\right)$ as $n \rightarrow \infty$, in $\mathrm{A}$, for every $k \in \mathbf{N}$,

( $\Delta$ convergence) a sequence $\left(h_{n}\right)$ in $\beta$ is said to be $\Delta$ convergent to $h$ in $\beta$, denoted by $h_{n} \stackrel{\Delta}{\rightarrow} h$, if there exists a $\left(s_{n}\right) \in \Delta$ such that $\left(h_{n}-h\right) \odot s_{n} \in \mathrm{A}$, for all $n \in \mathbf{N}$, and $\left(h_{n}-h\right) \odot s_{n} \rightarrow 0$ as $n \rightarrow \infty$ in A. For further discussion see [17-21].

\section{The Ultra-Boehmian Space $\beta_{s_{+}}$}

Denote by $\mathrm{D}_{+}$, or $\mathrm{D}\left(\mathbf{R}_{+}\right)$, the Schwartz space of $\mathbb{C}^{\infty}$ functions of bounded support. Let $\Delta_{+}$be the family of sequences $\left(s_{n}\right) \epsilon$ $\mathrm{D}\left(\mathbf{R}_{+}\right)$such that the following holds:

$$
\begin{aligned}
& \left(\Delta_{1}\right) \int_{\mathbf{R}_{+}} s_{n}(x) d x=1, \text { for all } n \in \mathbf{N}, \\
& \left(\Delta_{2}\right) s_{n}(x) \geq 0 \text {, for all } n \in \mathbf{N}, \\
& \left(\Delta_{3}\right) \operatorname{supp} s_{n} \subset\left(0, \varepsilon_{n}\right), \varepsilon_{n} \rightarrow 0 \text { as } n \rightarrow \infty .
\end{aligned}
$$

It is easy to see that each $\left(s_{n}\right)$ in $\Delta_{+}$forms a delta sequence. 
Let $f \in \mathcal{S}_{+}^{\prime}\left(\mathscr{L}^{r}, \alpha,\left(a_{i}\right), a\right)$ and $\sigma \in \mathrm{D}\left(\mathbf{R}_{+}\right)$be related by the expression:

$$
(f \cdot \sigma) v=f(\sigma \circledast v)
$$

where

$$
(\sigma \circledast v)(x)=\int_{\mathbf{R}_{+}} \sigma(t) v(x t) d t
$$

for every $v \in \mathcal{S}_{+}\left(\mathscr{L}^{r}, \alpha,\left(a_{i}\right), a\right)$.

Lemma 6. Let $f \in \mathcal{S}_{+}^{\prime}\left(\mathscr{L}^{r}, \alpha,\left(a_{i}\right), a\right)$ and $\sigma \in \mathrm{D}_{+}$and then $f \cdot \sigma \in \mathcal{S}_{+}^{\prime}\left(\mathscr{L}^{r}, \alpha,\left(a_{i}\right), a\right)$.

Proof. Using the weak topology of $\mathcal{S}_{+}^{\prime}\left(\mathscr{L}^{r}, \alpha,\left(a_{i}\right), a\right)$, we write

$$
|(f \cdot \sigma) v|=|f(\sigma \circledast v)| \leq C\|\sigma \circledast v\|_{r, a},
$$

where $v \in \mathcal{S}_{+}\left(\mathscr{L}^{r}, \alpha,\left(a_{i}\right), a\right)$. Hence, to complete the proof, we are merely required to show that $\sigma \circledast v \in \mathcal{S}_{+}\left(\mathscr{L}^{r}, \alpha,\left(a_{i}\right), a\right)$.

First, if $\sigma \in \mathrm{D}_{+}$and $v \in \mathcal{S}_{+}\left(\mathscr{L}^{r}, \alpha,\left(a_{i}\right), a\right)$, then choosing a compact set $\mathbf{K}$ containing the support of $\sigma$ yields

$$
\begin{aligned}
& \frac{(\sigma \circledast v)(x+\Delta x)-(\sigma \circledast v)(x)}{\Delta x} \\
& =\int_{\mathbf{R}_{+}} \sigma(t) \frac{v((x+\Delta x) t)-v(x t)}{\Delta x} d t
\end{aligned}
$$

which is dominated by $\sigma(t)\left|\mathscr{D}_{x} v(x)\right|$. The dominated convergence theorem and the principle of mathematical induction implies

$$
\mathscr{D}_{x}^{k}(\sigma \circledast v)=\sigma \circledast \mathscr{D}_{x}^{k} v
$$

Finally

$$
\begin{aligned}
\int_{\mathbf{R}_{+}}\left|\mathscr{D}^{k}(\sigma \circledast v)(x)\right|^{r} d x & =\int_{\mathbf{R}_{+}}\left|\left(\sigma \circledast \mathscr{D}^{k} v\right)(x)\right|^{r} d x \\
& \leq \int_{\mathbf{R}_{+}} \int_{\mathbf{K}}\left|\sigma(t) d t \mathscr{D}^{k} v(x t)\right|^{r} d x \\
& \leq M \int_{\mathbf{R}_{+}}\left|\mathscr{D}^{k} v(x t)\right|^{r} d x .
\end{aligned}
$$

Therefore

$\|\sigma \circledast v\|_{r, a} \leq d\|v\|_{r, a}<d a^{\alpha} a_{\alpha}$ for some constant $d$.

Thus $\sigma \circledast v \in \mathcal{S}_{+}\left(\mathscr{L}^{r}, \alpha,\left(a_{i}\right), a\right)$. This completes the proof of the lemma.

Lemma 7. Let $f_{1}, f_{2} \in \mathcal{S}_{+}^{\prime}\left(\mathscr{L}^{r}, \alpha,\left(a_{i}\right), a\right)$ and $\sigma \in \mathrm{D}_{+}$and then

(i) $\alpha f \cdot \sigma=\alpha(f \cdot \sigma), \alpha \in \mathbb{C}$,

(ii) $\left(f_{1}+f_{2}\right) \cdot \sigma=f_{1} \cdot \sigma_{1}+f_{2} \cdot \sigma_{2}$.

Proof of the above Lemma is obvious.
Lemma 8. Let $f_{n}, f \in \mathcal{S}_{+}^{\prime}\left(\mathscr{L}^{r}, \alpha,\left(a_{i}\right), a\right)$ and $\sigma \in \mathrm{D}_{+}$and then

$$
f_{n} \cdot \sigma \longrightarrow f \cdot \sigma \text {. }
$$

Proof. Let $v \in \mathcal{S}_{+}^{\prime}\left(\mathscr{L}^{r}, \alpha,\left(a_{i}\right), a\right)$ and then $\sigma \circledast v \in$ $\mathcal{S}_{+}^{\prime}\left(\mathscr{L}^{r}, \alpha,\left(a_{i}\right), a\right)$. Therefore

$$
\left(f_{n} \cdot \sigma-f \cdot \sigma\right) v=\left(\left(f_{n}-f\right) \cdot \sigma\right) v=\left(f_{n}-f\right)(\sigma \circledast v) .
$$

Hence $f_{n} \cdot \sigma \rightarrow f \cdot \sigma$ as $n \rightarrow \infty$.

Lemma 9. Let $f \in \mathcal{S}_{+}^{\prime}\left(\mathscr{L}^{r}, \alpha,\left(a_{i}\right), a\right)$ and $\left(s_{n}\right) \in \Delta_{+}$then

$$
f \cdot s_{n} \longrightarrow f \quad \text { in } \mathcal{S}_{+}^{\prime}\left(\mathscr{L}^{r}, \alpha,\left(a_{i}\right), a\right) \quad \text { as } n \longrightarrow \infty .
$$

Proof. Let $v \in \mathcal{S}_{+}\left(\mathscr{L}^{r}, \alpha,\left(a_{i}\right), a\right)$ and supp $s_{n} \subset\left(0, \varepsilon_{n}\right), n \in \mathbf{N}$ and then

$$
\left(f \cdot s_{n}\right) v=f\left(s_{n} \circledast \sigma\right) .
$$

It is sufficient to establish that $s_{n} \circledast v \rightarrow v$ as $n \rightarrow \infty$. By using (27) and $\Delta_{1}$ imply that

$$
\begin{aligned}
\int_{\mathbf{R}_{+}}\left|\mathscr{D}_{x}^{k}\left(s_{n} \circledast v-v\right)(x)\right|^{r} d x \\
\quad=\int_{\mathbf{R}_{+}}\left|\left(s_{n} \circledast \mathscr{D}_{x}^{k} v-\mathscr{D}_{x}^{k} v\right)(x)\right|^{r} d x \\
\quad=\int_{\mathbf{R}_{+}}\left|\int_{0}^{\varepsilon_{n}} s_{n}(t)\left(\mathscr{D}_{x}^{k} v(x t)-\mathscr{D}_{x}^{k} v(x)\right) d t\right|^{r} d x .
\end{aligned}
$$

Hence, the mean value theorem implies

$$
\begin{aligned}
& \int_{\mathbf{R}_{+}} \mid\left.\mathscr{D}_{x}^{k}\left(s_{n} \circledast v-v\right)(x)\right|^{r} d x \\
& \quad \leq \int_{\mathbf{R}_{+}}\left|\int_{0}^{\varepsilon_{n}} \xi s_{n}(t) \mathscr{D}_{x}^{k+1} v(x \xi) d t\right|^{r} d x,
\end{aligned}
$$

$\xi \in(0, t)$. Let $A_{1}=\sup _{s \in \mathbf{K}}\left|\mathscr{D}_{x}^{k+1} \psi(s)\right|$, where $\mathbf{K}$ is certain compact set. The calculations show that

$$
\left\|s_{n} \circledast v-v\right\|_{r, a} \leq F \varepsilon_{n} \longrightarrow 0 \quad \text { as } n \longrightarrow \infty,
$$

where $F$ is certain constant. Hence Lemma 9. The Boehmian space $\beta_{s_{+}}$is therefore constructed.

\section{6. $\beta_{\mathrm{Z}}$ and the Krätzel Transform of Ultra-Boehmians}

Denote by $\mathrm{Z}\left(\mathbf{R}_{+}\right)$, or $\mathrm{Z}_{+}$, the space of functions which are Krätzel transforms of ultradistributions in $\mathcal{S}_{+}^{\prime}\left(\mathscr{L}^{r}, \alpha,\left(a_{i}\right), a\right)$, and then convergence on $\mathrm{Z}_{+}$can be defined in such away that $E_{n} \rightarrow E_{*}$ in $Z_{+}$if $f_{n} \rightarrow f \in \mathcal{S}_{+}^{\prime}\left(\mathscr{L}^{r}, \alpha,\left(a_{i}\right), a\right)$ as $n \rightarrow \infty$, where $E_{n}=K_{v}^{p} f_{n}$ and $E_{*}=K_{v}^{p} f$. Let $E \in \mathrm{Z}_{+}$and $\sigma \in \mathrm{D}_{+}$and then it is proper to define

$$
(E \odot \sigma)(u)=\int_{\mathbf{R}_{+}} E(u t) \sigma(t) d t
$$

for each $u \in \mathbf{R}_{+}$. 
Lemma 10. Let $f \in \mathcal{S}_{+}^{\prime}\left(\mathscr{L}^{r}, \alpha,\left(a_{i}\right), a\right)$ and $\sigma \in \mathrm{D}_{+}$and then $K_{v}^{p}(f \cdot \sigma)=K_{v}^{p} f \odot \sigma$.

Proof. Let $\mathbf{K}$ be a compact set containing the support of $\sigma$, and then from (18) it follows that

$$
\begin{aligned}
K_{v}^{p}(f \cdot \sigma)(u) & =\left\langle(f \cdot \sigma)(y), \mathrm{Z}_{p}^{v}(u y)\right\rangle \\
& =\left\langle f(y),\left\langle\sigma(t), \mathrm{Z}_{p}^{v}((u t) y)\right\rangle\right\rangle \\
& =\int_{\mathbf{R}_{+}}\left\langle f(y), \mathrm{Z}_{p}^{v}((u t) y)\right\rangle \sigma(t) d t=K_{v}^{p} f \odot \sigma .
\end{aligned}
$$

Hence the lemma follows.

Lemma 11. Let $E \in \mathrm{Z}_{+}$and $\sigma \in \mathrm{D}_{+}$and then $E \odot \sigma \in \mathrm{Z}_{+}$.

Proof. $E \in \mathrm{Z}_{+}$implies $K_{v}^{p} f=E$, for some $f \in$ $\mathcal{S}_{+}^{\prime}\left(\mathscr{L}^{r}, \alpha,\left(a_{i}\right), a\right)$. Hence $E \odot \sigma=K_{v}^{p} f \odot \sigma=K_{v}^{p}(f \cdot \sigma) \epsilon$ $\mathrm{Z}_{+}$.

The following are lemmas which can be easily proved by the aid of the corresponding lemmas from the previous section. Detailed proof is avoided. First, if ${K_{v}{ }^{p}}^{-1}$ is the inverse Krätzel transform of $K_{v}^{p}$, and then

Lemma 12. Let $E \in \mathrm{Z}_{+}$and $\sigma \in \mathrm{D}_{+}$and then

$$
K_{v}^{-1}(E \odot \sigma)=K_{v}^{-1} E \cdot \sigma .
$$

Lemma 13. Let $E_{1}, E_{2} \in \mathrm{Z}_{+}$and then for all $\sigma_{1}, \sigma_{2} \in \mathrm{D}_{+}$we have

(1) $\left(E_{1}+E_{2}\right) \odot \sigma_{1}=E_{1} \odot \sigma_{1}+E_{2} \odot \sigma_{1}$,

(2) $(a E) \odot \sigma_{1}=a\left(E \odot \sigma_{1}\right)$.

Lemma 14. Let $E_{n} \rightarrow E$ and $\left(s_{n}\right) \in \Delta_{+}$and then $E_{n} \odot s_{n} \rightarrow$ E.

Lemma 15. Let $E_{n} \rightarrow E$ and $\sigma \in \mathrm{D}_{+}$and then $E_{n} \odot \sigma \rightarrow E \odot \sigma$.

With the previous analysis, the Boehmian space $\beta_{\mathrm{z}_{+}}$is constructed. The sum of two Boehmians and multiplication by a scalar in $\beta_{\mathrm{z}_{+}}$is defined in a natural way $\left[f_{n} / \phi_{n}\right]+$ $\left[g_{n} / \psi_{n}\right]=\left[\left(\left(f_{n} \odot \psi_{n}\right)+\left(g_{n} \odot \phi_{n}\right)\right) /\left(\phi_{n} \odot \psi_{n}\right)\right]$ and $\alpha\left[f_{n} / \phi_{n}\right]=$ $\left[\alpha\left(f_{n} / \phi_{n}\right)\right], \alpha \in \mathbb{C}$.

The operation $\odot$ and the differentiation are defined by

$$
\left[\frac{f_{n}}{\phi_{n}}\right] \odot\left[\frac{g_{n}}{\psi_{n}}\right]=\left[\frac{f_{n} \odot g_{n}}{\phi_{n} \odot \psi_{n}}\right], \quad \mathscr{D}^{k}\left[\frac{f_{n}}{\phi_{n}}\right]=\left[\frac{\mathscr{D}^{k} f_{n}}{\phi_{n}}\right] .
$$

With the aid of Lemma 10 we define the extended Krätzel transform of a Boehmian $\left[f_{n} / s_{n}\right] \in \beta_{s_{+}}$to be a Boehmian in $\beta_{z_{+}}$expressed by the relation:

$$
\overrightarrow{\mathbf{K}}_{v}^{p}\left[\frac{f_{n}}{s_{n}}\right]=\left[\frac{K_{v}^{p} f_{n}}{s_{n}}\right]
$$

Lemma 16. $\overrightarrow{\mathbf{K}}_{v}^{p}: \beta_{s_{+}} \rightarrow \beta_{\mathrm{z}_{+}}$is well defined and linear mapping.

Proof is a straightforward conclusion of definitions.

Definition 17. Let $\left[E_{n} / s_{n}\right] \in \beta_{\mathrm{z}_{+}}$and then the inverse of $\overrightarrow{\mathbf{K}}$ is defined as follows:

$$
\overrightarrow{\mathbf{K}}_{v}^{p}\left[\frac{E_{n}}{s_{n}}\right]=\left[\frac{K_{v}^{p} E_{n}}{s_{n}}\right]
$$

for each $\left(s_{n}\right) \in \Delta_{+}$.

Lemma 18. $\overrightarrow{\mathbf{K}}_{v}^{p}$ is an isomorphism from $\beta_{s_{+}}$into $\beta_{\mathrm{Z}_{+}}$.

Proof. Assume $\overrightarrow{\mathbf{K}}_{v}^{p}\left[f_{n} / s_{n}\right]=\overrightarrow{\mathbf{K}}_{v}^{p}\left[g_{n} / t_{n}\right]$, then it follows from (41) and the concept of quotients of two sequences $K_{v}^{p} f_{n}$ ○ $t_{m}=K_{v}^{p} g_{m} \odot s_{n}$. Therefore, Lemma 10 implies $K_{v}^{p}\left(f_{n} \cdot t_{m}\right)=$ $K_{v}^{p}\left(g_{m} \cdot \phi_{n}\right)$. Employing properties of $K_{v}^{p}$ implies $f_{n} \cdot t_{m}=$ $g_{m} \cdot s_{n}$. Thus, $\left[f_{n} / s_{n}\right]=\left[g_{n} / t_{n}\right]$. Next we establish that $\overrightarrow{\mathbf{K}}_{v}^{p}$ is onto. Let $\left[K_{v}^{p} f_{n} / s_{n}\right] \in \beta_{\mathrm{z}_{+}}$be arbitrary and then $K_{v}^{p} f_{n} \odot$ $s_{m}=K_{v}^{p} f_{m} \odot s_{n}$ for every $m, n \in \mathbf{N}$. Hence $f_{n}, f_{m} \in$ $\delta_{+}^{\prime}\left(\mathscr{L}^{r}, \alpha,\left(a_{i}\right), a\right)$ are such that $K_{v}^{p}\left(f_{n} \cdot s_{m}\right)=K_{v}^{p}\left(f_{m} \cdot s_{n}\right)$. Hence the Boehmian $\left[f_{n} / s_{n}\right] \in \beta_{s_{+}}$satisfies the equation $\overrightarrow{\mathbf{K}}_{v}^{p}\left[f_{n} / s_{n}\right]=\left[K_{v}^{p} f_{n} / s_{n}\right]$.

This completes the proof of the lemma.

Lemma 19. Let $\left[E_{n} / s_{n}\right] \in \beta_{\mathrm{z}_{+}}, E_{n}=K_{v}^{p} f_{n}$, and $\phi \in \mathrm{D}_{+}$and then

$$
\begin{aligned}
\overrightarrow{\mathbf{K}}_{v}^{p} & \left(\left[\frac{E_{n}}{s_{n}}\right] \odot \phi\right)=\left[\frac{E_{n}^{-1}}{s_{n}}\right] \cdot \phi, \\
& \overrightarrow{\mathbf{K}}_{v}^{p}\left(\left[\frac{f_{n}}{s_{n}}\right] \bullet \phi\right)=\left[\frac{E_{n}}{s_{n}}\right] \odot \phi .
\end{aligned}
$$

Proof. It follows from (42) that

$$
\overrightarrow{\mathbf{K}}_{v}^{-1}\left(\left[\frac{E_{n}}{s_{n}}\right] \odot \phi\right)=\overrightarrow{\mathbf{K}}_{v}^{p}\left(\left[\frac{E_{n} \odot \phi}{s_{n}}\right]\right)=\left[\frac{K_{v}^{-1}\left(E_{n} \odot \phi\right)}{s_{n}}\right] .
$$

Applying Lemma 12 leads to

$$
\overrightarrow{\mathbf{K}}_{v}^{p}\left(\left[\frac{E_{n}}{s_{n}}\right] \odot \phi\right)=\left[\frac{K_{v}^{-1} E_{n}}{s_{n}}\right] \cdot \phi=\left[\frac{E_{n}^{-1}}{s_{n}}\right] \cdot \phi .
$$

Proof of the second part is similar. This completes the proof of the lemma.

Theorem 20. $\overrightarrow{\mathbf{K}}_{v}^{p}: \beta_{s_{+}} \rightarrow \beta_{\mathrm{Z}_{+}}$and $\overrightarrow{\mathbf{K}}_{v}^{p}: \beta_{\mathrm{Z}_{+}} \rightarrow \beta_{s_{+}}$are continuous with respect to $\delta$ and $\Delta$ convergences.

Proof. First of all, we show that $\overrightarrow{\mathbf{K}}_{v}^{p}: \beta_{s_{+}} \rightarrow \beta_{\mathrm{z}_{+}}$and $\overrightarrow{\mathbf{K}}_{v}{ }^{p}$ : $\beta_{\mathrm{z}_{+}} \rightarrow \beta_{s_{+}}$are continuous with respect to $\delta$ convergence. Let $\beta_{n} \stackrel{\delta}{\rightarrow} \beta$ in $\beta_{s_{+}}$as $n \rightarrow \infty$ and then we establish that 
$\overrightarrow{\mathbf{K}}_{v}^{p} \beta_{n} \rightarrow \overrightarrow{\mathbf{K}}_{v}^{p} \beta$ as $n \rightarrow \infty$. In view of [10], there are $f_{n, k}$ and $f_{k}$ in $\mathcal{S}_{+}^{\prime}\left(\mathscr{L}^{r}, \alpha,\left(a_{i}\right), a\right)$ such that

$$
\beta_{n}=\left[\frac{f_{n, k}}{s_{k}}\right], \quad \beta=\left[\frac{f_{k}}{s_{k}}\right]
$$

such that $f_{n, k} \rightarrow f_{k}$ as $n \rightarrow \infty$ for every $k \in \mathbf{N}$. The continuity condition of the Krätzel transform implies $E_{n, k} \rightarrow$ $E_{k}$ as $n \rightarrow \infty, E_{n, k}=K_{v}^{p} f_{n, k}$, and $E_{k}=K_{v}^{p} f_{k}$ in the space $\mathrm{Z}_{+}$. Thus, $\left[E_{n, k} / s_{k}\right] \rightarrow\left[E_{k} / s_{k}\right]$ as $n \rightarrow \infty$ in $\beta_{\mathrm{z}_{+}}$.

To prove the second part of the lemma, let $g_{n} \stackrel{\delta}{\rightarrow} g \in$ $\beta_{\mathrm{Z}_{+}}$as $n \rightarrow \infty$. From [10], we have $g_{n}=\left[E_{n, k} / s_{k}\right]$ and $g=\left[E_{k} / s_{k}\right]$ for some $E_{n, k}, E_{k} \in Z_{+}$where $E_{n, k} \rightarrow E_{k}$ as $n \rightarrow \infty$. Hence $K_{v}^{-1} E_{n, k} \rightarrow K_{v}^{-1} E_{k}$ in $\beta_{s_{+}}$as $n \rightarrow \infty$. That is, $\left[E_{n, k}^{-1} / s_{k}\right] \rightarrow\left[E_{k}^{-1} / s_{k}\right]$ as $n \rightarrow \infty$. Using (42) we get $\overrightarrow{\mathbf{K}}_{v}^{p}\left[E_{n, k} / s_{k}\right] \rightarrow \overrightarrow{\mathbf{K}}_{v}^{-1}\left[E_{k} / s_{k}\right]$ as $n \rightarrow \infty$.

Now, we establish continuity of $\overrightarrow{\mathbf{K}}_{v}^{p}$ and $\overrightarrow{\mathbf{K}}_{v}^{p}$ with respect to $\Delta_{+}$convergence. Let $\beta_{n} \stackrel{\Delta}{\rightarrow} \beta$ in $\beta_{s_{+}}$as $n \rightarrow \infty$. Then, we find $f_{n} \in \mathcal{S}_{+}^{\prime}\left(\mathscr{L}^{r}, \alpha,\left(a_{i}\right), a\right)$, and $\left(s_{n}\right) \in \Delta_{+}$such that $\left(\beta_{n}-\beta\right)$. $s_{n}=\left[\left(f_{n} \cdot s_{k}\right) / s_{k}\right]$ and $f_{n} \rightarrow 0$ as $n \rightarrow \infty$. Employing (41) we get

$$
\overrightarrow{\mathbf{K}}_{v}^{p}\left(\left(\beta_{n}-\beta\right) \cdot s_{n}\right)=\left[\frac{K_{v}^{p}\left(f_{n} \cdot s_{k}\right)}{s_{k}}\right] .
$$

Hence, from (41) and Lemma 19 we have $\overrightarrow{\mathbf{K}}_{v}^{p}\left(\left(\beta_{n}-\beta\right) \cdot s_{n}\right)=$ $\left[\left(E_{n} \odot s_{k}\right) / s_{k}\right]=E_{k} \rightarrow 0$ as $n \rightarrow \infty$ in $Z_{+}$. Therefore

$$
\begin{aligned}
& \overrightarrow{\mathbf{K}}_{v}^{p}\left(\left(\beta_{n}-\beta\right) \cdot s_{n}\right) \\
& \quad=\left(\overrightarrow{\mathbf{K}}_{v}^{p} \beta_{n}-\overrightarrow{\mathbf{K}}_{v}^{p} \beta\right) \odot s_{n} \longrightarrow 0 \text { as } n \longrightarrow \infty .
\end{aligned}
$$

Hence, $\overrightarrow{\mathbf{K}}_{v}^{p} \beta_{n} \stackrel{\Delta}{\rightarrow} \overrightarrow{\mathbf{K}}_{v}^{p} \beta$ as $n \rightarrow \infty$. Finally, let $g_{n} \stackrel{\Delta}{\rightarrow} g$ in $\beta_{\mathrm{z}_{+}}$ as $n \rightarrow \infty$ and then we find $E_{k} \in Z_{+}$such that $\left(g_{n}-g\right) \odot s_{n}=$ $\left[\left(E_{k} \odot s_{k}\right) / s_{k}\right]$ and $E_{k} \rightarrow 0$ as $n \rightarrow \infty$ for some $\left(s_{n}\right) \in \Delta_{+}$ and $E_{k}=K_{v}^{p} f_{n}$.

Next, using (42), we obtain $\overrightarrow{\mathbf{K}}_{v}^{-1}\left(\left(g_{n}-g\right) \odot s_{n}\right)=\left[K_{v}^{-1}\left(E_{k} \odot\right.\right.$ $\left.\left.s_{k}\right) / s_{k}\right]$. Lemma 19 implies that

$$
\begin{aligned}
& \overrightarrow{\mathbf{K}}_{v}^{p}\left(\left(g_{n}-g\right) \odot s_{n}\right) \\
& =\left[\frac{f_{n} \cdot s_{k}}{s_{k}}\right]=f_{n} \longrightarrow 0 \text { as } n \longrightarrow \infty \text { in } \mathcal{S}_{+}^{\prime}\left(\mathscr{L}^{r}, \alpha,\left(a_{i}\right), a\right) .
\end{aligned}
$$

Thus $\overrightarrow{\mathbf{K}}_{v}^{-1}\left(\left(g_{n}-g\right) \odot s_{n}\right)=\left(\overrightarrow{\mathbf{K}}_{v}^{p} g_{n}-\overrightarrow{\mathbf{K}}_{v}{ }^{p} g\right) \cdot s_{n} \rightarrow 0$ as $n \rightarrow \infty$. Hence, we have $\overrightarrow{\mathbf{K}}_{v}^{p} g_{n} \stackrel{\Delta}{\rightarrow} \overrightarrow{\mathbf{K}}_{v}^{p}$ g as $n \rightarrow \infty$ in $\beta_{s_{+}}$.

This completes the proof of the theorem.

\section{Acknowledgment}

The authors express their sincere thanks to the referees for the careful and detailed reading of the earlier version of paper and the very helpful suggestions that improved the paper substantially. The second author also acknowledges that this project was partially supported by University Putra Malaysia under the ERGS Grant Scheme with Project no. 5527068.

\section{References}

[1] E. Krätzel, "Integral transformations of Bessel-type," in Generalized Functions and Operational Calculus (Proc. Conf., Varna, 1975), pp. 148-155, Bulgarian Academy of Sciences, Sofia, Bulgaria, 1979.

[2] E. Krätzel and H. Menzer, "Verallgemeinerte HankelFunktionen," Publicationes Mathematicae Debrecen, vol. 18, pp. 139-147, 1971.

[3] A. H. Zemanian, Distribution Theory and Transform Analysis, Dover Publications, New York, NY, USA, 2nd edition, 1987.

[4] D. I. Cruz-Báez and J. Rodríguez Expósito, "New inversion formulas for the Krätzel transformation," International Journal of Mathematics and Mathematical Sciences, vol. 25, no. 4, pp. 253-263, 2001.

[5] A. H. Zemanian, Generalized Integral Transformations, Dover Publications, New York, NY, USA, 2nd edition, 1987.

[6] J. A. Barrios and J. J. Betancor, "A Krätzel's integral transformation of distributions," Collectanea Mathematica, vol. 42, no. 1, pp. 11-32, 1991.

[7] B. Fisher and A. Kiliçman, "A commutative neutrix product of ultradistributions," Integral Transforms and Special Functions, vol. 4, no. 1-2, pp. 77-82, 1996.

[8] C. Roumieu, "Ultra-distributions définies sur $R^{n}$ et sur certaines classes de variétés différentiables," Journal d'Analyse Mathématique, vol. 10, pp. 153-192, 1963.

[9] C. Roumieu, "Sur quelques extensions de la notion de distribution," Annales Scientifiques de l'École Normale Supérieure. Troisième Série, vol. 77, pp. 41-121, 1960.

[10] H. Komatsu, "Ultradistributions. I. Structure theorems and a characterization," Journal of the Faculty of Science. University of Tokyo. Section IA. Mathematics, vol. 20, pp. 25-105, 1973.

[11] A. Beurling, Quasi-Analiticity and Generalized Distributions, Lectures 4 and 5, A. M. S. Summer Institute, Stanford, Calif, USA, 1961.

[12] R. D. Carmichael, R. S. Pathak, and S. Pilipović, "Cauchy and Poisson integrals of ultradistributions," Complex Variables. Theory and Application, vol. 14, no. 1-4, pp. 85-108, 1990.

[13] R. S. Pathak, Integral Transforms of Generalized Functions and Their Applications, Gordon and Breach Science Publishers, Amsterdam, The Netherlands, 1997.

[14] R. S. Pathak, "A distributional generalised Stieltjes transformation," Proceedings of the Edinburgh Mathematical Society. Series II, vol. 20, no. 1, pp. 15-22, 1976.

[15] S. K. Q. Al-Omari, "Certain class of kernels for Roumieu-type convolution transform of ultra-distributions of compact support," Journal of Concrete and Applicable Mathematics, vol. 7, no. 4, pp. 310-316, 2009.

[16] S. K. Q. Al-Omari, "Cauchy and Poison integrals of tempered ultradistributions of Roumieu and Beurling types," Journal of Concrete and Applicable Mathematics, vol. 7, no. 1, pp. 36-46, 2009.

[17] T. K. Boehme, “The support of Mikusiński operators," Transactions of the American Mathematical Society, vol. 176, pp. 319-334, 1973. 
[18] S. K. Q. Al-Omari, D. Loonker, P. K. Banerji, and S. L. Kalla, "Fourier sine (cosine) transform for ultradistributions and their extensions to tempered and ultraBoehmian spaces," Integral Transforms and Special Functions, vol. 19, no. 5-6, pp. 453-462, 2008.

[19] P. Mikusiński, "Fourier transform for integrable Boehmians," The Rocky Mountain Journal of Mathematics, vol. 17, no. 3, pp. 577-582, 1987.

[20] P. Mikusiński, "Tempered Boehmians and ultradistributions," Proceedings of the American Mathematical Society, vol. 123, no. 3, pp. 813-817, 1995.

[21] R. Roopkumar, "Mellin transform for Boehmians," Bulletin of the Institute of Mathematics. Academia Sinica. New Series, vol. 4, no. 1, pp. 75-96, 2009. 


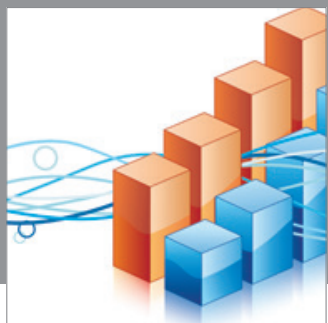

Advances in

Operations Research

mansans

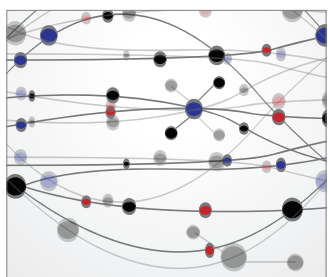

The Scientific World Journal
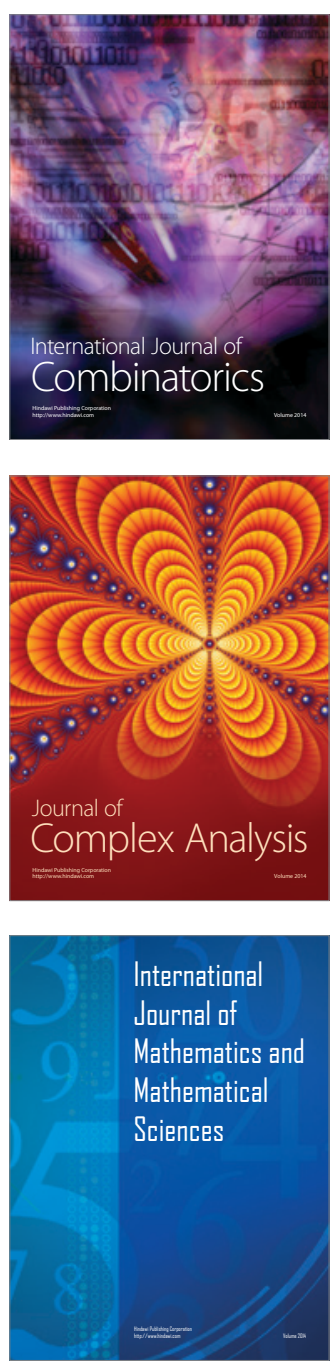
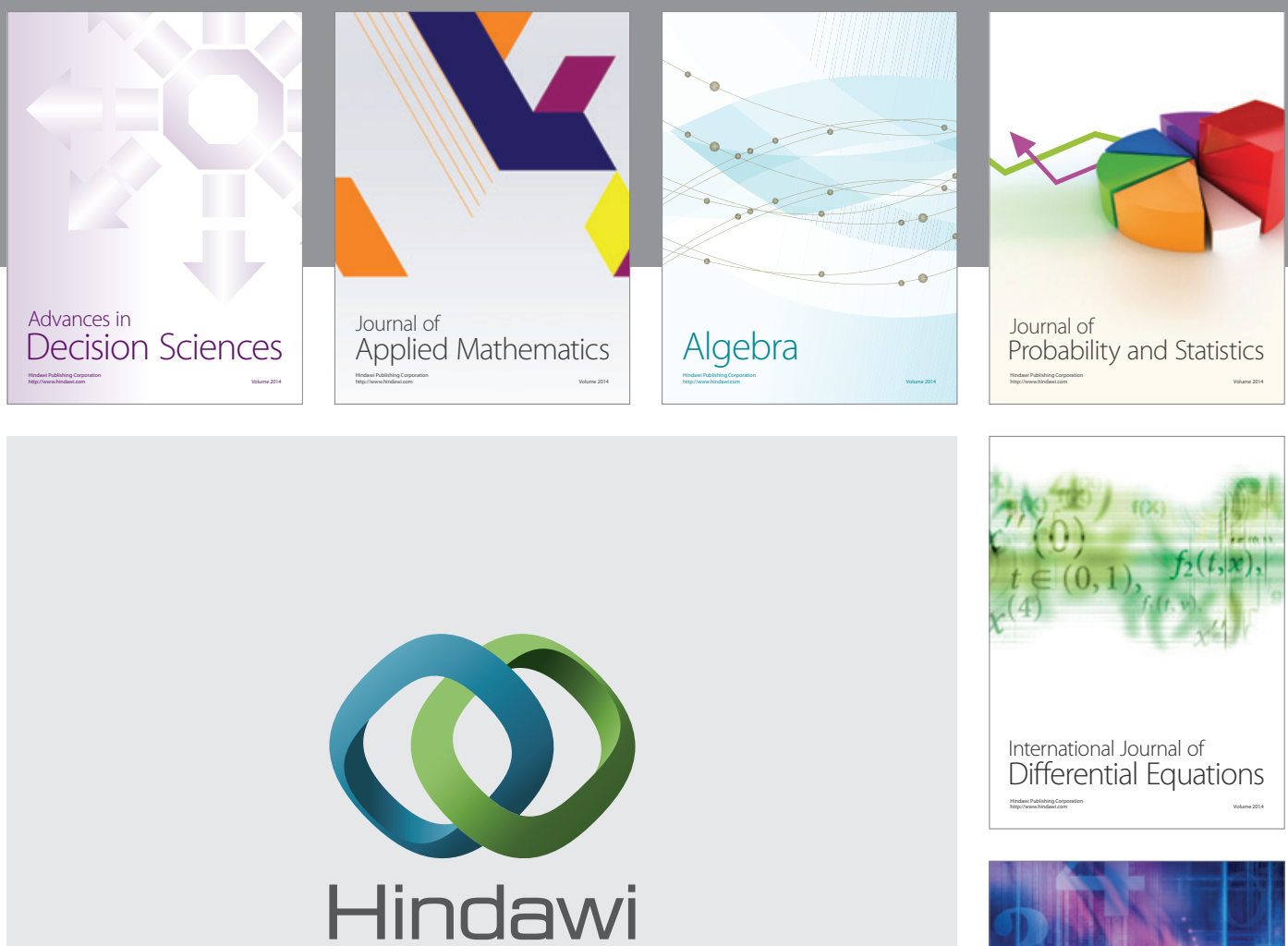

Submit your manuscripts at http://www.hindawi.com
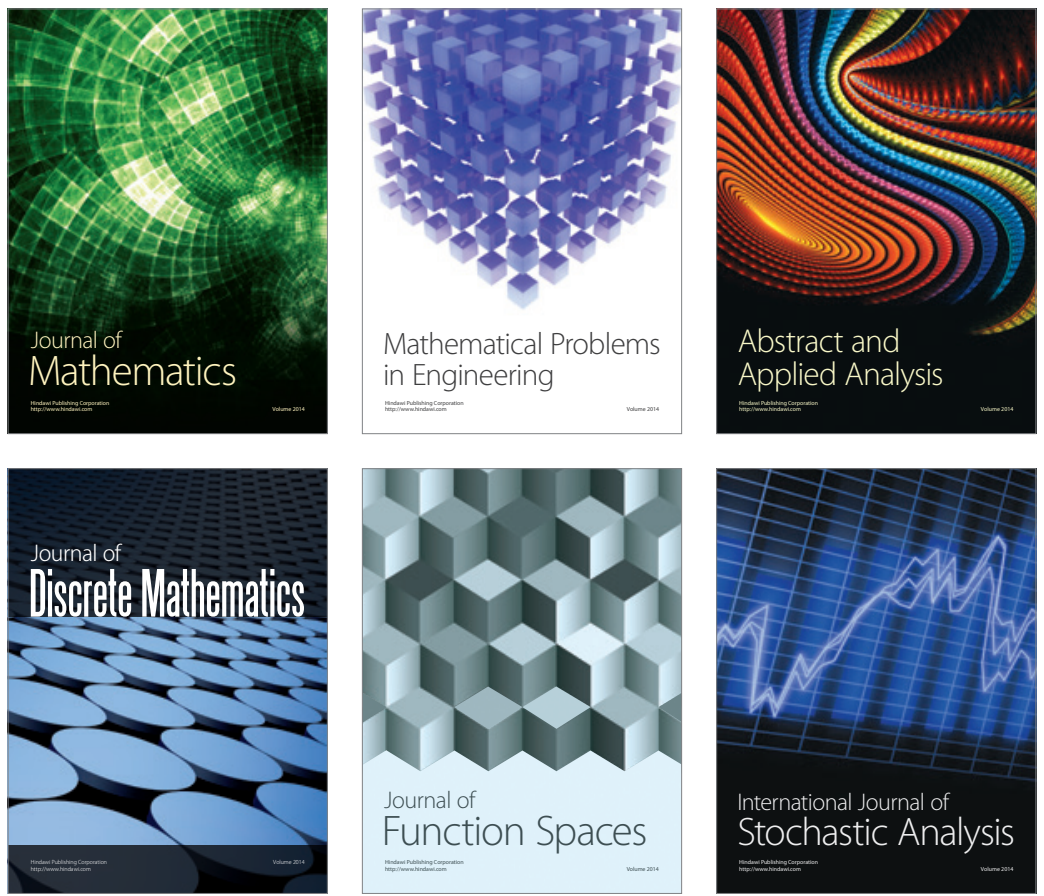

Journal of

Function Spaces

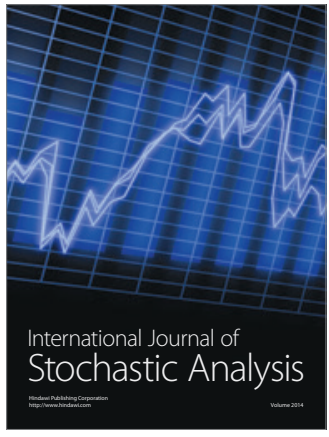

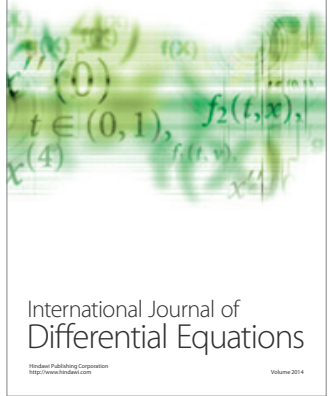
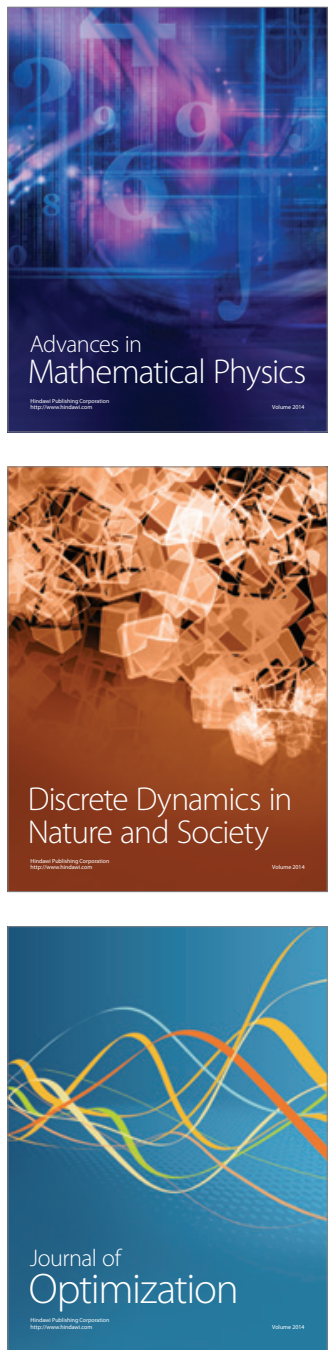